\title{
Distributed measurement of vibrations in a ramified fiber structure using phase sensitive optical time domain reflectometry and wavelength routing concepts
}

\author{
Juan Pastor Graells, Hugo F. Martins, Sonia Martin-Lopez and Miguel Gonzalez-Herraez \\ Department of Electronics, University of Alcalá, Escuela Politécnica Superior DO-231, Alcalá de Henares 28805, Spain
}

\begin{abstract}
We show the possibility of fully distributed measurement of vibrations in a complex fiber topology using phase-sensitive optical time-domain reflectometry (ФOTDR) and wavelength routing. This could enable the monitoring of complex structures showing ramifications. OCIS codes: (60.2300); (60.2370); (120.7280);
\end{abstract}

\section{Introduction}

Distributed fiber optic sensing technology offers clear advantages over conventional point sensors when the number of points to be analyzed along a structure reaches several hundreds or thousands. Typically, distributed sensors are well suited for the monitoring of long linear structures like railways, pipelines, high-power electric lines, etc. However, there is few or no report on the distributed interrogation of complex ramified structures such as gas or oil distribution networks, smart grids, cable bridges, etc. The monitoring of complex ramified structures without an increase in sensing heads could be beneficial in some applications requiring e.g. cost-effective monitoring of several branches attached to a main line. This work aims at providing a convenient solution to this demand. In particular, we show that wavelength routing concepts can be conveniently used in combination with a distributed sensor to interrogate this type of topologies.

Phase-sensitive optical time-domain reflectometry (ФOTDR) is a powerful technique that allows the fully distributed monitoring of vibrations along an optical fiber cable. Monitoring of intrusions over large perimeters is an important application of this technique [1]. Unlike traditional OTDRs (which use incoherent light and therefore can only measure intensity variations along the fiber), in a phase-sensitive OTDR (TOTDR), a pulse of highly coherent light is injected into a conventional single-mode fiber and the light reflected from different scattering centers interferes coherently to produce the detected optical power trace [2-5]. Because the position of these scattering centers is random, the $\Phi O T D R$ trace typically has random oscillations. This random pattern remains constant if the scattering centers do not suffer any change. However, in the case of localized vibrations, the trace shows variations synchronized with the vibration frequency. Due to its potential for fully distributed measurement of vibrations, TOTDR attracted considerable attention for more than two decades [6]. Intrusion monitoring over large perimeters has become a major field of application of this technology given its high sensitivity and long range.

Up to now, OOTDRs have been used for monitoring vibrations along a unique fiber optic line. In this work, we present a technique to perform fully distributed measurement of vibrations over ramified structures. It is based on the use of intelligent wavelength routing over the selected fiber topology. Our development can have very interesting applications in smart grids and energy distribution networks in general.

\section{Experimental Setup}

The experimental setup used in this work (Fig 1) is composed by a typical ФOTDR scheme as developed in [2] and a set of five fiber spools of standard telecommunication fiber with different lengths. These fibers are connected by suitable wavelength routing filters (Dense Wavelength Division Multiplexers, DWDMs) simulating a branched architecture of a smart grid as depicted in Fig.1 (FUT1-5). The distributed feedback laser (DFB) within the ФOTDR is temperature and current-controlled to provide coherent light pulses at different wavelengths. Depending on the exact wavelength used, the TOTDR pulses are routed to one fiber or the other, and the backscatter trace is obtained for each one of them over a period of 1 second. After 1 second, the vibrations over each fiber are analyzed as a function of the position, using the conventional methodology [2].

A highly coherent tunable laser diode (LD) (linewidth of $1.6 \mathrm{MHz}$ ) emitting around $1547 \mathrm{~nm}$ was used as the light source (the LD is driven by a standard current and temperature controller). The current and temperature controls are essential to achieve the desired routing of the probe pulses as explained above. An isolator has been used to avoid possible reflections being directed to the laser, which could affect the laser stability. The output 
wavelength of the laser is tuned continuously to sweep all the wavelength values necessary to have access to all the fiber branches.

A semiconductor optical amplifier (SOA), with rise/fall times in the order $2.5 \mathrm{~ns}$, driven by a waveform signal generator $(\mathrm{SG})$, is used to create $50 \mathrm{~ns}$ almost square pulses. The spatial resolution of the system is determined basically by the duration of the pulse [6], therefore it is $5 \mathrm{~m}$ in this case. Between the signal pulses, the SOA is negatively biased so as to enhance the extinction ratio (ER) of the delivered pulses. An ER of $>50 \mathrm{~dB}$ can be achieved this way. In this configuration, the ER has a very high impact in the SNR of the detected trace [2].

An EDFA is used to boost the power of the TOTDR pulses (up to $200 \mathrm{~mW}$ ) before sending them into the fiber. In order to minimize the effect of the amplified spontaneous emission (ASE) added by the erbium-doped fiber amplifier (EDFA), we insert a tunable fiber Bragg grating (FBG) filter working in reflection after the EDFA. The spectral profile is the typical spectrum of a $100 \%$ reflection FBG, and its spectral width is $0.8 \mathrm{~nm}$. Before being coupled into the fiber under test (FUT), light passes through an attenuator, which allows varying the input power in the fiber.

Different ramifications have been created with five fiber spools (FUT1, FUT2, FUT3, FUT4 and FUT5), with lengths of $10 \mathrm{~km}, 200 \mathrm{~m}, 300 \mathrm{~m}, 4.5 \mathrm{~km}$ and $1 \mathrm{~km}$, respectively. DWDM filters (with a spectral width of $0.8 \mathrm{~nm}$ ) were used to connect the spools and create the proper wavelength routing scheme to address each branch. Thus, the different possibilities of interrogating the structure are B1 (FUT1+FUT3), associated to a wavelength of 1547.89 $\mathrm{nm}$, B3 (FUT1+FUT2+FUT5), associated to $1548.86 \mathrm{~nm}$, and B2 (FUT1+FUT2+FUT4) which can be interrogated with any $\mathrm{C}$-band wavelength other than those used in the other two channels.

The signal back reflected from the fiber is amplified (using an EDFA) and then goes through a tunable optical filter with a spectral width of $1 \mathrm{~nm}$, centered on the wavelength used at the measurement. The signal was recorded with a $125 \mathrm{MHz}$ photodetector and a high-speed digitizer.

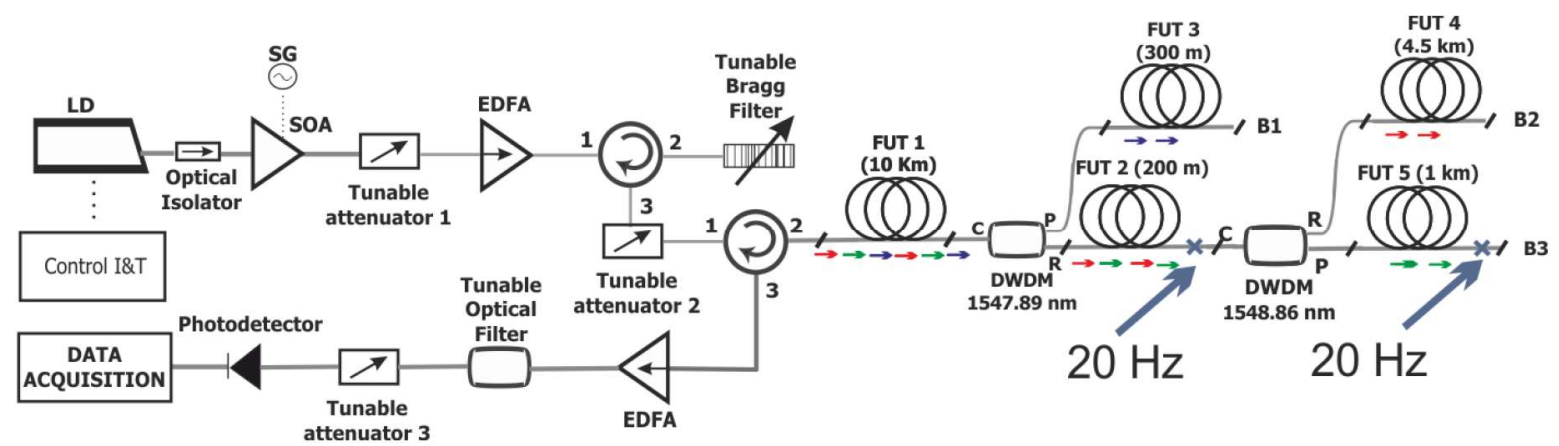

Fig.1. Experimental Setup. Acronyms are explained in the text.

To simulate vibrations over the structure, we apply a shaker in two positions of the ramified fiber structure at the same time. Typical vibrations that cause potential threats to smart grids are usually in the order of tens of $\mathrm{Hz}$ (e.g. heavy machinery), therefore two vibrations of $20 \mathrm{~Hz}$ were applied on the fiber under test in two different points, as it is shown in Fig.1. The positions where the shaker is applied are chosen (at the end of FUT 2 and at the end of FUT 5) so that using the B1 combination $(1547.89 \mathrm{~nm})$ there is no visible vibration, while using the B2 combination we see one vibration (only at the end of FUT 2) and finally, using the B3 combination (1548.86 nm) we observe two vibrations at the expected positions (one at the end of FUT 2 and one at the end of FUT 5).

\section{Results}

The three different branches have been measured varying laser wavelength as it is observed in Fig. 1. Thus, as mentioned, B1 is not perturbed, B2 is perturbed in only one point and B3 is perturbed in two points. In Fig. 2 the optical power and a measure of the acoustic energy versus distance are depicted. In Fig.2 (a) - top it is represented the case in which the laser was tuned at $1547.89 \mathrm{~nm}$. As it can be observed, the constituent fibers of branch 1 (B1) can be clearly identified (FUT $110 \mathrm{~km}$, FUT 3, $300 \mathrm{~m}$ ). In the representation of acoustic energy at $20 \mathrm{~Hz}$ vs distance (Fig. 2(b) - top), we can see that in this case no vibration is recorded as expected.

In Fig.2 (a) - middle, the constituent fibers of branch 2 (B2) can be clearly identified (FUT $110 \mathrm{~km}$, FUT 2, 200 $\mathrm{m}$, and FUT 4, $4.5 \mathrm{~km}$ ). In Fig. 2(b)- middle we can now see a vibration of $20 \mathrm{~Hz}$ localized at $10200 \mathrm{~m}$ from the input point. Finally, branch 3 (B3) was observed using wavelength of $1548.86 \mathrm{~nm}$. Again, the constituent fibers of 
branch 3 (B3) can be clearly identified (FUT $110 \mathrm{~km}$, FUT 2, $200 \mathrm{~m}$, and FUT 5, $1 \mathrm{~km}$ ) can be clearly identified in the optical trace (Fig 2(a) - bottom). Two vibrations of $20 \mathrm{~Hz}$ can be localized in this case, one at the end of B3 (11200 $\mathrm{m}$ far from the beginning) and the other one at the same point than before (10200 $\mathrm{m}$ of B2 which is common to $10200 \mathrm{~m}$ of B3). The energy registered on the first position is higher than the one of the second position due to the decrease of signal power after the DWDM. It should be noted that neither the FUT 3 nor the FUT 4 were detected by the interrogation unit as can be observed in Fig.2(a) bottom, as in the other cases we indeed observed no measurable crosstalk with the other fiber combinations.

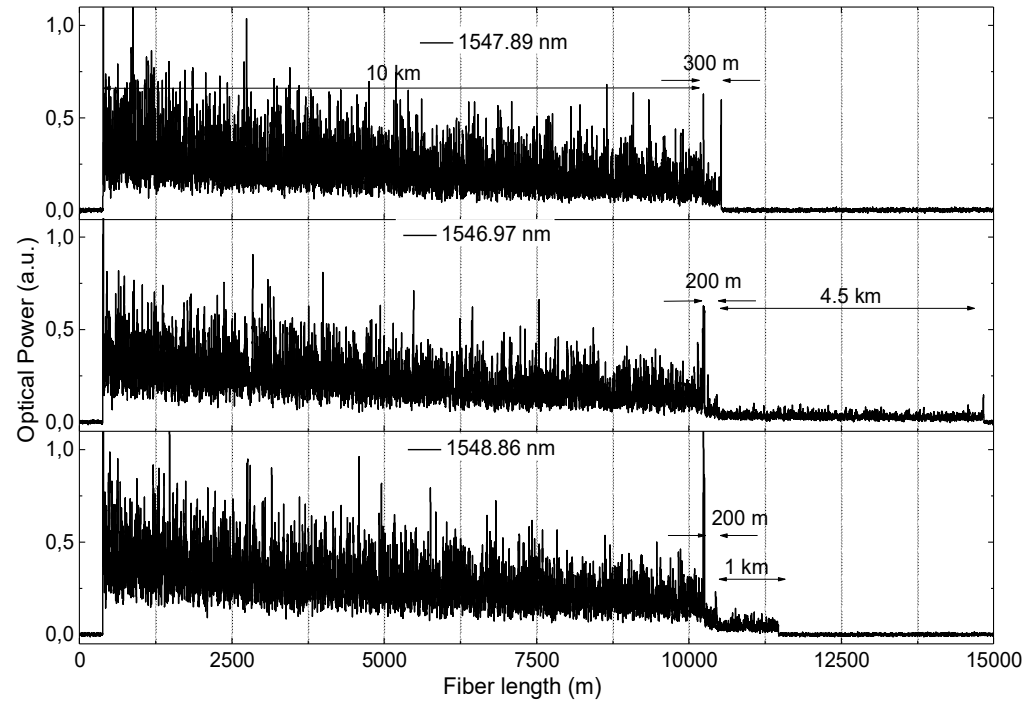

(a)

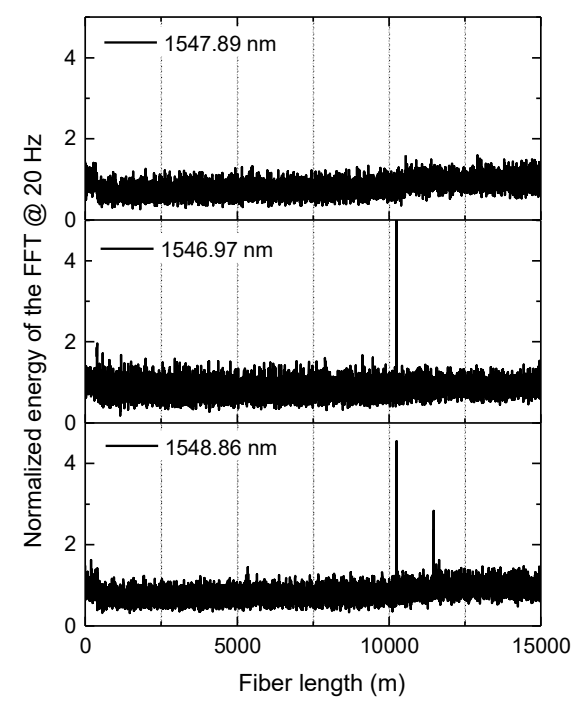

(b)

Fig. 2. Optical Power along the fiber (a) and Acoustic Energy around $20 \mathrm{~Hz}$ along the fiber (b).

\section{Conclusions}

In conclusion, we have shown that wavelength routing concepts can be conveniently used in combination with a distributed sensor to perform a fully distributed interrogation of this type of complex fiber topologies. In particular, we have presented a technique to perform fully distributed measurement of vibrations over ramified structures using ФOTDR and wavelength routing. The idea has been experimentally demonstrated over a range not exceeding $15 \mathrm{~km}$ with 5 meter spatial resolution and 2 ramifications ( 3 possible signal routes). It is envisioned that our development may have very interesting applications in smart grids and energy distribution networks in general.

This work was supported by funding from the European Research Council through Starting Grant U-FINE (Grant no. 307441) and the Spanish "Plan Nacional de I+D+i" through project TEC2012-37958-C02-01 and the INTERREG SUDOE program ECOAL-MGT. The work of S. Martín-López was supported by the Spanish Ministry of Science and Innovation through a "Ramón y Cajal" Contract.. H. Martins acknowledge a scholarship from FCT Fundação para a Ciência e a Tecnologia (Portuguese Foundation for Science and Technology), SFRH / BD / 76991 / 2011.

\section{References}

[1] J. C. Juarez, E. W. Maier, K. N. Choi, and H. F. Taylor, "Distributed fiber-optic intrusion sensor system", J. Lightw. Technol., 23, 2081-2087 (2005).

[2] H. F. Martins, S. Martin-Lopez, P. Corredera, M. L. Filograno, O. Frazão, and M. Gonzalez-Herraez, "Coherent Noise Reduction in High Visibility Phase-Sensitive Optical Time Domain Reflectometer for Distributed Sensing of Ultrasonic Wave”, J. Lightw. Technol 31, 3631-3637 (2013).

[3] J. C. Juarez and H. F. Taylor, "Field test of a distributed fiber-optic intrusion sensor system for long perimeters", Appl Optics, 46, 1968-1971 (2007).

[4] Z. Qin, L. Chen and X. Bao, "Wavelet Denoising Method for Improving Detection Performance of Distributed Vibration Sensor", IEEE Photonic Tech L, 24, 542-544 (2012).

[5] Y. Koyamada, M. Imahama, K. Kubota and K. Hogari, "Fiber-Optic Distributed Strain and Temperature Sensing With Very High Measurand Resolution Over Long Range Using Coherent OTDR", J Lightwave Technol, 27, 1142-1146 (2009).

[6] H.F. Taylor and C. E. Lee, “Apparatus and method for fiber optic intrusion sensing", Texas A \& M University System, College Station, TX, USA, U. S. Patent 5194847, Mar. 16, 1993. 